\title{
Towards a functional label set for the online dictionary of Flemish Sign Language
}

\author{
Caro Brosens (VGTC, Expertise Center for Flemish Sign Language) \\ caro.brosens@vgtc.be \\ Hannes De Durpel (VGTC, Expertise Center for Flemish Sign Language) \\ hannes.de.durpel@vgtc.be \\ Inez Beukeleers (KULeuven) \\ Beatrijs Wille (Ghent University) \\ inez.beukeleers@kuleuven.be \\ Beatrijs.Wille@ugent.be
}

\begin{abstract}
How do we know when, with whom and in what context to use a particular sign? Most traditional explanatory dictionaries of oral languages provide clarifying labels indicating the connotation and/or usage of specific words, e.g. archaic, neologism, offensive, euphemism, etc. These clarifying labels are, however, lacking in most sign language lexicographic research.

In a continuing effort to improve online sign dictionaries, the expertise center for Flemish Sign Language (the VGTC) is currently conducting preliminary research towards a detailed label system and accompanying methodology suited for sign languages.

The objective of this project is two-fold: 1) adapting our electronic lexical database and the online dictionary to make displaying these labels possible, and 2) compiling a practical set of labels primarily suited to Flemish Sign Language (VGT), as well as detailing a functional methodology to determine when (and which) labels of the aforementioned label set should be allocated to which signs. For the latter, a combination of corpus-based analysis and community-based approaches is proposed.

This exploratory study has contributed to our wider understanding of label sets and their use, and it has reopened the discussion of language- and modality-specific labels.
\end{abstract}

Keywords: Flemish Sign Language, usage information, usage labels, register labels

\section{Introduction}

How do we know when, with whom and in what context to use a particular sign? Most traditional explanatory dictionaries of oral languages provide clarifying labels indicating the connotation and/or usage of specific words, e.g. archaic, offensive, euphemism, etc. These clarifying labels are not yet established, and thus lacking, in most sign language lexicographic research. This dissimilarity between signed and oral language lexicographic research can be mainly attributed to the relatively young age of sign languages and their research. In the early stages, lexicographic work often resulted in something closer to a glossary rather than a full-fledged dictionary (Zwitserlood 2010). These early "dictionaries" were, in practice, only useful for hearing people trying to communicate with Deaf people. 
In the past decade, however, online dictionaries for sign languages have become more widely accessible. New technological advancements have opened up a whole new world of possibilities - that is, digital dictionaries are no longer restricted in space/volume and it has become technologically possible to compile and analyze a signed language corpus (McKee and McKee 2013). This opens up new possibilities in meeting the needs and wants of native signers, thus broadening the target audience of the dictionary.

A similar evolution can be observed in Flanders. The growing demand for a Flemish Sign Language (VGT) dictionary in the 1990s led to small lexicographic projects all over Flanders starting in 1999. At that time, special care was taken to avoid the influence of Dutch: only native signers were present at these recorded data gathering sessions and the data was elicited through lists of concepts rather than Dutch words (Vermeerbergen and Van Herreweghe 2018). The lexicographic data compiled in these sessions led to the launch of the first online dictionary VGT - Dutch in 2004. This was a bilingual and bidirectional dictionary, where users could search for a sign by entering its handshape, location or movement, or by typing a Dutch word (Vermeerbergen and Van Herreweghe 2018). In 2012 the expertise center for Flemish Sign Language (the VGTC) took over the maintenance of the dictionary.

After over a decade of use, it became apparent that, due to advancements in technology, the 2004 dictionary required updating. In 2018, the VGTC therefore applied for, and received, a one-time grant to thoroughly renew the dictionary. Besides formal modernization, i.e., layout, structural changes were made to the dictionary to ensure the website is responsive to all screen sizes. Even though there were other search options, the alphabetical list of Dutch words stood out most in the previous dictionary. In the new design the videos, the signs themselves, now have a more prominent place. This was further enhanced by updating the video recordings. Searching from VGT to Dutch is now no longer done through SignWriting, but through images of the parameters, which makes the search function more user-friendly. Furthermore, semantic categories or domain labels were added, which allows users to also search thematically. A new feedback feature allows users to give feedback in written Dutch and in VGT. In the fall of 2019, the current dictionary was launched. ${ }^{1}$

Another important step in the modernization process was the implementation of Signbank. Signbank is an electronic lexical database specifically developed to collect lexicographic data for signed languages. Since sign languages are very visual in nature, it is important to use a database which allows for easy uploading and processing of videos. This system also offers the possibility to annotate signs morphologically, phonologically and semantically. It has been in use at the VGTC since 2017 as a central hub for all lexicographic data on VGT and it directly feeds the online dictionary. In other words: Signbank is used both as a database and as a dictionary writing system.

In a continuing attempt to improve online sign dictionaries and more fully utilize the available technological possibilities, the VGTC is currently conducting preliminary research towards a detailed label system and accompanying methodology suited for sign languages.

The objective of this one-year project is two-fold:

1. The technical aspect: To utilize the technological possibilities to the fullest, both Signbank and the online dictionary will have to be adjusted. Signbank already allows for labels, but the ones it currently contains are only used internally. A second set of labels will be added which can be made publicly available on the dictionary. The VGT dictionary will first need to be adapted before it can display these labels publicly.

1. The current VGT - Dutch / Dutch - VGT dictionary can be found at https://woordenboek.vlaamsegebarentaal.be/ 
2. The research aspect: A practical set of labels primarily suited to Flemish Sign Language (VGT) is being created. The idea is that this set should be generalizable to all signed languages and their dictionaries. Another part is to detail a functional methodology to determine when (and which) labels of the aforementioned label set should be allocated to a certain sign.

This article will focus primarily on the latter aspect.

\section{Theoretical background}

Usage labels provide the dictionary users with valuable metalinguistic information about "the domain of application of a certain definition" (Verkuyl, Janssen, and Jansen 2003, 2). This information should not be equated with the definition because labels restrict the definition further without being part of it (Verkuyl, Janssen, and Jansen 2003).

Hitherto there is no consensus among lexicographers on how to categorize these labels (Namatende-Sakwa 2011; Bakema 1994). Stachurska (2018) gives a summary of several different approaches and points out that a useful distinction has been made between group labels and register labels (Stachurska 2018; Verkuyl, Janssen, and Jansen 2003). Group labels indicate who or which group tends to use the lexeme. This category can be further divided into four smaller categories: geographic labels (e.g., Belgian Dutch, British English, American English, regional/dialect), temporal labels (e.g., old-fashioned, archaic, neologism), frequency labels (e.g., rare), and domain labels (e.g., medicine, education, nature, ...). Some online sign language dictionaries already contain geographic or domain labels. Only very few online sign language dictionaries contain both, three examples are the Flemish Sign Language (VGT), French Belgian Sign Language (LSFB), and Australian Sign Language (Auslan) dictionaries. As previous research in Flanders has focused on studying regional variation (De Weerdt et al. 2003; Vermeerbergen and Van Herreweghe 2018), on identifying hiatuses in the dictionary (ibid.), and on developing and implementing the labels obtained in our VGT dictionary (Vermeerbergen and Van Herreweghe 2018), the current research project explores the use of register labels. These labels indicate the register of the lexeme, i.e., the label indicates which communicative situation the lexeme tends to be used in. Some examples of register labels are vulgar, (in)formal, biblical, and poetic/literary. So far, very few online sign language dictionaries - for example that of New Zealand Sign Language ${ }^{2}$ - contain register labels. Out of their label set of five labels, three are group labels (neologism, archaic and rare), and two are register labels (informal, and obscene). Interestingly, the group labels used in this dictionary are not yet observed in other sign language dictionaries.

When comparing different dictionaries, it quickly becomes apparent that there is significant variety among different label sets. Since there is no consensus on what an ideal label set looks like; every dictionary has its own approach. This variety brings to light a number of potential pitfalls.

The first one is incomplete and unbalanced taxonomies. For instance, if the label Middle English contains a sub label Eastern Middle English, one might wonder about the absence of labels like Northern/Southern/Western Middle English. Granted, sometimes this would lead to labels that are illogical or that serve no purpose. However, an incomplete taxonomy might also indicate an unsystematic approach (Bakema 1994). Since the two biggest types of group labels can already be found in the VGT dictionary, that is geographic and domain labels,

2. The online dictionary of New Zealand Sign Language can be found at https://www.nzsl.nz/ 
incomplete taxonomies are not the biggest concern in this project. However, it remains important to be aware of any potential incompleteness.

Secondly, due to the number of labels and lexemes, labels are often not applied consistently or systematically (Antón Ortego 2011; Bakema 1994). For example, some names of surgeries are labeled surgery or surgical while for others its hypernym medical is used. This indicates again how important it is to keep the label set as balanced and straightforward as possible. It also shows the need for a clear agreement and mutual understanding between all members of the team on the scope and definition of each label. By restricting our label set and working with several researchers so that all data is revised several times, we hope to minimize this risk.

Lastly, not (or inconsistently) defining the meaning of a label for the users is a problem. It is not self-evident that dictionary users just happen to know what a usage label is, what these labels mean, and how to interpret them (Namatende-Sakwa 2011). Not every dictionary explains or discloses the scope of their labels, or how these are used in that specific dictionary. Many dictionaries do not even provide an overview of their entire label set (NamatendeSakwa 2011; Bakema 1994). For example, when several labels are included with similar or synonymous meanings, is it not always clear if those labels are used interchangeably or if there is an underlying logic in the distinctions. This can unintentionally indicate linguistic differences that are not there (Bakema 1994, 12). In other words, not properly defining the labels can be confusing and can make them lose their value for the user. To mitigate this, the VGT dictionary will be updated with an additional information page including a legend with the meaning and the scope of all the labels, both in VGT and in written Dutch.

Besides these pitfalls, we identified several other aspects in labeling usage information that warrant special attention.

To begin with, a label should be noticeable and not abbreviated. Because of technological advances, dictionaries are no longer bound by a limited storage capacity. Where paper copies required brevity, digital copies are in theory unlimited (McKee and McKee 2013). This allows for usage information to be incorporated into longer descriptions or definitions (Verkuyl, Janssen, and Jansen 2003). As users frequently skip labels, for instance because they are often inconspicuous and abbreviated, it might be advisable to do so (Van der Meer 2008). In this way, more users can be reached.

Sign languages do not have a script the way spoken languages do. The writing systems that do exist, e.g. SignWriting, are not widely used or known. Because of this, most sign language data are presented in the form of videos. So far, very few dictionaries of signed languages offer definitions, let alone a definition in the sign language being documented. Even if a signed definition is available, it is safe to assume that users would not always watch it fully-in which case, the user information might not reach them. Therefore, it seems advisable to include both a clear, unabbreviated label and information in the definition (if provided) in signed language dictionaries.

Secondly, connotation spans a continuum. Besides neutral (which carries no label because it is the default), all aspects of the continuum should be accounted for. "When negative connotations are indicated, it is advisable to also add positive labels like humorous or (term of) endearment." (Frieda Steurs, scientific director of the Dutch Language Institute, personal communication, 15/12/2020). Looking at lemmata that are labeled as sensitive or offensive, it appears that they usually refer to humans or animals. Yet connotations regarding inanimate objects also should be considered. Referring to a house as a hovel might be hurtful to the owner, but the more telling aspect is the negative mental imagery it evokes. This is where the labels disapproving or pejorative and approving or ameliorative, as seen for ex- 
ample in the Oxford Advanced Learners Dictionary, come into play. The VGT dictionary label set should also reflect the entire continuum.

Subsequently, when naming labels, it is important to strike a balance between conciseness/accurateness and clearness. A lexicographer needs to consider whether terms like $p e$ jorative or neologism are transparent enough for the average user of their dictionary. If there is a more simple, more common alternative, it seems best to opt for it to avoid confusion.

Even if the intention is to merely describe the language, with labels - and in particular register labels - the underlying opinion of the lexicographer will often shine through (Namatende-Sakwa 2011). When a lexeme is described as offensive, it is implied it would be best not to use it. Labeling sex related words as informal rather than obscene, implicitly shows the lexicographer's perspective.

The next thing to contemplate is whether there is a link with the surrounding spoken language. When it comes to lexicography, spoken languages are leaps and bounds ahead of signed languages. Can usage information of lexemes in the surrounding spoken language help get a clearer picture of how certain signs are used? How does the relation between majority and minority language, their differences in status, and their geographical closeness affect register in the minority language? Further research will be needed before a definitive answer can be formulated. Regardless, critical evaluation will most likely always be crucial.

Finally, corpus analysis will be another difficult issue to navigate. Spoken language corpora often contain extensive and varied natural language data, which can then be used to determine by whom, and in which registers, a certain word tends to be used. According to linguistic researcher Rob Tempelaars, the Dutch Language Institute (INT) indeed relies on its impressive corpus to allocate labels to entries in their General Dutch Dictionary (ANW): "An experienced lexicographer can usually deduce from a given context what the connotation of a word or phrase is." (Personal communication, 15/12/2020).

In sign linguistics, corpus analysis is still in its infancy. Compared to spoken language corpora, there is just not as much natural language data available (yet). Furthermore, getting reliable and consistent annotations of an entire sign corpus is an endeavor with many obstacles that takes years if not decades. Using corpus analysis is often not financially viable or practically feasible. The team responsible for one of the few sign language dictionaries with register labels, that of NZSL, also indicates that their corpus is not (yet) suitable for determining the register of signs. Instead, they allocate labels by verification within their own team as well as with a group of experts (Mireille Vale, database manager, personal communication, 28/01/2021).

In the last decade, much care, time, and resources have been invested in compiling the VGT corpus. ${ }^{3}$ This corpus consists of 5 TB or 140 hours of video data produced by 120 deaf L1 signers (Over het corpus). Despite the size of the corpus, it cannot yet be consistently used for analysis since only part of it has been annotated so far and these annotations are not yet efficiently searchable. At this point, about 40 hours of all the data have been transcribed (Wille et al., in preparation). Nonetheless, the value of the VGT corpus should not be underestimated. It should be used as much as possible in gathering lexicographic data on VGT. The VGTC is therefore currently working on linking corpus annotations to Signbank.

These points of special interest were kept in mind throughout the project.

3. For more information on the VGT Corpus see: http://www.corpusVGT.be. 


\section{Methodology}

Thanks to a thorough literature review and a close investigation of several dictionaries, two main methodological challenges were identified. First of all, the label set should fully suit the modality-specific needs of signed languages. Secondly, the natural use of lexical signs should be examined in order to allocate the right label(s). In what follows, these two components will be further explained.

\subsection{Compiling a label set for signed languages}

Several reasons make compiling such a label set more challenging. First of all, the set has to be built from scratch. There is practically no literature so far, as hardly any online dictionaries of sign languages already contain register labels. As mentioned earlier, the amount of variety between existing label sets and their, at first sight, lack of a logical or systematic approach makes it difficult to get a clear grip on the label landscape as a whole. Secondly, while some traditional labels are clearly irrelevant for most sign languages (e.g. written, biblical), for other labels it is still unclear if and how to apply them to sign languages (e.g. ironic, sarcastic). Even though some research has been conducted regarding the comprehension of sarcasm and irony by prelingually deaf people (for example González-Cuenca and Linero 2020; Li et al. 2019), a deep understanding of how sarcasm and irony present in sign language still seems far away, with only a few studies published on the subject (for instance Mantovan, Giustolisi, and Panzeri 2019). Worth noting here is existing research into euphemisms (Sze, Wei, and Wong 2017), and research into politeness (Roush 2011; Hoza 2007). Both provide interesting early insights into register, connotation and face-saving strategies in signed discourse which have not yet been researched for VGT. Lastly, traditional labels used in oral language dictionaries will need to be supplemented with labels specific to sign languages. While developing these, there is always a risk of undetected gaps. Working closely with native signers during this project, as well as having native signers on our team, hopefully allowed us to minimize this risk.

To get an overview of possible labels, all style and attitude labels of four dictionaries were gathered (see Table 1). These dictionaries were selected because: 1) they provide the user with an overview of their label set and 2) they represent a wide range of different types of dictionaries. The four consulted dictionaries were:

- Online dictionary of NZSL = online trilingual (NZSL - English - Maori) bimodal translation dictionary

- Algemeen Nederlands Woordenboek (General Dutch Dictionary) or ANW = online monolingual dictionary for native speakers

- Oxford Advanced Learners Dictionary = monolingual print dictionary for L2-learners

- Van Dale (Dutch - English) = bilingual translation print dictionary

In compiling the label set for VGT, this list was used as a rough draft. This initial set was then adapted as follows (see Table 2). For the sake of clarity and conciseness, some labels indicating a different level/intensity of the same concept were combined. So instead of several labels like informal and slang, one umbrella term was adopted. Labels denoting the same concept that use a different term in different dictionaries, remain as one label. Other labels like "written" are excluded because of irrelevance. The number of occurrences 
Table 1: A compilation of labels occurring in four pre-identified dictionaries. The labels followed by a $(*)$ occur under (slightly) different names in different dictionaries.

\begin{tabular}{|c|c|c|c|c|}
\hline & ODNZSL & ANW & OALD & Van Dale NL - EN \\
\hline very formal & & $\checkmark$ & & \\
\hline formal & & $\checkmark$ & $\checkmark$ & $\checkmark$ \\
\hline informal & $\checkmark$ & $\checkmark$ & $\checkmark$ & $\checkmark$ \\
\hline very informal $\left.{ }^{*}\right)$ & & $\checkmark$ & $\checkmark$ & $\checkmark$ \\
\hline obscene $(*)$ & $\checkmark$ & $\checkmark$ & $\checkmark$ & $\checkmark$ \\
\hline biblical & & $\checkmark$ & & \\
\hline religious & & $\checkmark$ & & \\
\hline poetic & & $\checkmark$ & & $\checkmark$ \\
\hline literary & & $\checkmark$ & $\checkmark$ & \\
\hline jargon $\left(^{*}\right)$ & & $\checkmark$ & $\checkmark$ & \\
\hline approving $\left(^{*}\right)$ & & & $\checkmark$ & \\
\hline disapproving $(*)$ & & $\checkmark$ & $\checkmark$ & $\checkmark$ \\
\hline eufemistic & & $\checkmark$ & & $\checkmark$ \\
\hline (term of) endearment & & $\checkmark$ & & \\
\hline humorous $\left(^{*}\right)$ & & $\checkmark$ & $\checkmark$ & $\checkmark$ \\
\hline ironic & & $\checkmark$ & $\checkmark$ & $\checkmark$ \\
\hline sarcastic & & $\checkmark$ & & \\
\hline insulting & & $\checkmark$ & $\checkmark$ & $\checkmark$ \\
\hline hurtful & & $\checkmark$ & $\checkmark$ & \\
\hline (very) frequent & & $\checkmark$ & & \\
\hline rare & $\checkmark$ & $\checkmark$ & & $\checkmark$ \\
\hline old-fashioned & & $\checkmark$ & $\checkmark$ & $\checkmark$ \\
\hline $\operatorname{archaic}(*)$ & $\checkmark$ & $\checkmark$ & $\checkmark$ & $\checkmark$ \\
\hline neologism & $\checkmark$ & $\checkmark$ & & \\
\hline literally & & & & $\checkmark$ \\
\hline figurative & & & $\checkmark$ & $\checkmark$ \\
\hline
\end{tabular}

of the labels in other label sets was not the only aspect taken into account. For example, neologisms are - for technical and practical reasons - only labelled in online dictionaries. Since the VGT dictionary is also online, it is only logical to also include it.

Some uses like irony or sarcasm are not yet well researched in sign languages. To allow time for additional research, by the VGTC and other researchers, these labels will be implemented in a later phase. The division of these labels into phases was based on two criteria: 1) usefulness and relevance of the usage labels to the local Deaf community and 2) feasibility.

While researching existing label sets is important, existing labels most likely need to be supplemented with labels specifically suited for sign languages and/or minority languages. One suggestion might be graphic to indicate (possible) offensiveness due to the visual nature or underlying motivation of the sign. Think for example of some very visual signs, which refer to a physical aspect or stereotype, like JEW, that have not remained unnoticed (Schreuer 2019). Another example is one of the signs for ATTIC in VGT, which refers to it as where people hang themselves. The term graphic is inspired by the common content warning in popular media which denotes possible shocking or offensive imagery. Most of the sign-specific labels identified during this project, however, are related to grammatical features or part of speech tagging and were not included in this label set. 
Table 2: Our primary label set.

\begin{tabular}{lll} 
Phase 1 & Phase 2 & Phase 3 \\
\hline offensive & formal & ironic \\
approving & informal & sarcastic \\
disapproving & obscene & (term of) endearment \\
neologism & euphemistic & humorous \\
old-fashioned & frequent & figuratively \\
graphic & rare & literally \\
& & affected by spoken language \\
& & affected by sign language \\
\hline
\end{tabular}

In the end it was decided not to include jargon because the VGT dictionary already contains domain labels which specify the field a certain sign belongs to.

Phase 3 mostly consists of labels that will be applicable to a sub-meaning or sub-definition of the lexeme. Except for the two labels about linguistic influence, these labels will not be applicable to an entry as a whole.

\subsection{Detecting the natural use of lexical signs}

Confirming which signs should be allocated to which labels is challenging because sign language lexicography cannot rely in the same way on corpus analysis. Other - more community based - approaches might be labor intensive. Furthermore, it is often hard to determine how reliable the results are and where to draw the line, because it relies on signers' intuitions. This will be the next stage of the current research.

In order to mitigate the downsides of the community-based approaches and to make up for the fact that corpus-based analysis is not yet an efficient option, a combination of both is proposed.

\subsubsection{Step one: identification}

First of all, the team will identify signs that might require one of the labels of the predefined label set. The already established domain labels will be used as a starting point, starting with the categories that will most likely contain signs which will need a given label, for instance graphic (domain: religion, sexuality, ...) or offensive (domain: religion, countries, society, sexuality, ...). After this, the more neutral categories can be examined.

\subsubsection{Step two: verification}

In this second step, the initial assumptions of the team will be formally verified through a combination of corpus analysis (when possible) and community sourcing, i.e., through discussing the signs with a carefully selected panel of proficient signers. Each participant considers VGT their native or primary language and they maintain close ties with the Deaf community. Having deaf family members is seen as a plus. Furthermore, all participants have demonstrated the ability to reflect on their own language use and that of the community.

This committee of experts is already in use to determine which signs belong in the dictionary and which do not. In other words, this committee helps determine if a certain sign 
that has been collected is produced correctly (if all the parameters are correct), if the scope of the possible translations is correct, and if it is used in their area/regional variant. In this panel each of the five Flemish provinces is represented. This approach allows the team to work closely with the community, and not have to rely solely on the internal expertise of our deaf researchers.

By supplementing the expertise of our team and the valuable data of the corpus with community sourcing, we hope to figure out the use of certain signs faster. The plan is to extend the scope of these in-depth conversations in the committee by adding discussions about the contexts and connotations of certain signs, thus going beyond the "do you know this sign?" and "is it used in your area?" questions.

\subsubsection{Step three: publication}

At this stage, all the collected information is brought together and evaluated by the team. The preliminary label will be added to the entry concerned in Signbank. If the preliminary label with regards to a specific entry gets rejected, the label as well as the reason for the rejection will only be stored with said entry in Signbank for internal use. Accepted labels with regards to a certain entry will be made publicly available in the online dictionary. Cases where no consensus is reached will be stored for internal use in Signbank until further research brings to light new information.

\subsubsection{Step four: following up}

Language changes and evolves; this means allocated labels need to be followed up on. Starting again from step one, the VGTC team will regularly go through all the signs with a given label and will identify signs that will need to be re-evaluated.

\section{Conclusion}

This exploratory study has contributed to our wider understanding of label sets and their use, and it has reopened the discussion of language and modality specific labels. Inspired by the label sets of four pre-identified dictionaries, a first practical label set has been compiled for the online VGT dictionary. In order to bypass technical and other methodological constraints regarding the allocation of the labels, a combination of two methods was proposed, namely corpus analysis and community sourcing. This methodological choice has enabled us to bring the study to a successful conclusion and allows us to move to the next step of identifying signs and integrating the labels into the online VGT dictionary.

\section{References}

Antón Ortego, María Teresa. 2011. "The usage of field labels in English-Spanish bilingual edictionaries from the perspective of translators." In Electronic Lexicography in the 21st Century New Applications for New Users Proceedings of eLex 2011, edited by Iztok Kosem and Karmen Kosem, 1547-1555. Bled: Trojína Institute for Applied Slovene Studies.

Bakema, Peter. 1994. “Connotatieve labels in Nederlandse woordenboeken.” De nieuwe taalgids 87 (1): 515-532. 
De Weerdt, Kristof, Eline Vanhecke, Mieke Van Herreweghe, and Myriam Vermeerbergen. 2003. De dovengemeenschap in Vlaanderen: Doorlichting, sensibilisering en standaardisering van de Vlaamse gebarentaal: Eindrapport, luik 2. Op onderzoek naar de Vlaamse gebarenschat. Gent: Fevlado-Diversus.

González-Cuenca, Antonia, and María José Linero. 2020. "Lies and irony understanding in deaf and hearing adolescents." The Journal of Deaf Studies and Deaf Education 25 (4): 517-529. https://doi.org/10.1093/deafed/enaa014.

Hoza, Jack. 2007. It's not what you sign, it's how you sign it: Politeness in American Sign Language. Washington, D.C.: Gallaudet University Press.

Li, Degao, Shaai Wang, Fan Zhang, Li Zhu, Tao Wang, and Xiaolu Wang. 2019. "DHH students' comprehension of irony in self-paced reading." The Journal of Deaf Studies and Deaf Education 24 (3): 270-279. https://doi.org/10.1093/deafed/enz009.

Mantovan, Lara, Beatrice Giustolisi, and Francesca Panzeri. 2019. "Signing something while meaning its opposite: The expression of irony in Italian Sign Language (LIS).” Journal of Pragmatics 142:47-61. https://doi.org/10.1016/j.pragma.2018.12.008.

McKee, Rachel Locker, and David McKee. 2013. "Making an Online Dictionary of New Zealand Sign Language.” Lexikos 23 (1). https://doi.org/10.5788/23-1-1227.

Namatende-Sakwa, Lydia. 2011. “Problems of usage labelling in English lexicography.” Lexikos 21 (21): 305-315.

Over het corpus. https://www.corpusvgt.be/.

Roush, Daniel R. 2011. "Language between bodies: A cognitive approach to understanding linguistic politeness in American Sign Language.” Sign Language Studies 11 (3): 329-374. https://doi.org/10.1353/sls.2011.0000.

Schreuer, Milan. 2019. “University Denounced for Showing Sign Language for 'Jewish' as a Hooked Nose." https: / www.nytimes.com/2019/09/20/world/ europe/belgium- signlanguage-jews.html.

Stachurska, Anna. 2018. "On the codification of usage by labels.” Journal of Language and Cultural Education 6 (1): 89-107. https://doi.org/10.2478/jolace-2018-0006.

Sze, Felix Y. B., Monica Xiao Wei, and Aaron Yiu Leung Wong. 2017. "Taboos and euphemisms in sex-related signs in Asian sign languages.” Linguistics 55 (1). https://doi.org/10.1515/ ling-2016-0034.

Van der Meer, Geart. 2008. "On connotation, denotation and all that, or: Why a nigger is not a "Black Person"." In Proceedings of the XIII EURALEX International Congress. 15-19 July 2008, edited by Elisenda Bernal and Janet DeCesaris, 1547-1555. Barcelona: Universitat Pompeu Fabra.

Verkuyl, Henk J., Maarten Janssen, and Frank Jansen. 2003. "The codification of usage by labels." In Terminology and Lexicography Research and Practice, edited by Piet vanSterkenburg, 6:297-311. Amsterdam: John Benjamins Publishing Company. 
Vermeerbergen, Myriam, and Mieke Van Herreweghe. 2018. "Looking back while moving forward: The impact of societal and technological developments on Flemish sign language lexicographic practices.” International Journal of Lexicography 31, no. 2 (June 1, 2018): 167-195. https://doi.org/10.1093/ijl/ecy008.

Wille, Beatrijs, Inez Beukeleers, Mieke Van Herreweghe, and Myriam Vermeerbergen. in preparation. "Big things often have small beginnings: on the development and use of small and big corpora for Flemish Sign Language linguistic research.”

Zwitserlood, Inge. 2010. "Sign language lexicography in the early 21st century and a recently published dictionary of Sign Language of the Netherlands." International Journal of Lexicography 23 (4): 443-476. https://doi.org/10.1093/ijl/ecq031. 